\title{
Static and quasistatic response of Ag surfaces to a uniform electric field
}

\author{
H. Ishida ${ }^{1}$ and A. Liebsch ${ }^{2}$ \\ ${ }^{1}$ College of Humanities and Sciences, Nihon University, Sakura-josui, Tokyo 156, Japan \\ ${ }^{2}$ Institut für Festkörperforschung, Forschungszentrum Jülich, 52425 Jülich, Germany
}

(Received 14 June 2002; published 23 October 2002)

\begin{abstract}
By performing a first-principles surface-embedded Green-function calculation, we study the static and quasistatic response of semi-infinite $\operatorname{Ag}(111),(001)$, and (110) surfaces to a uniform electric field oriented perpendicular to the surface. In addition to the linear and nonlinear induced electron densities in the static limit, we calculate the electron-hole pair excitation rate within the quasistatic approximation using a golden-rule formula. It will be shown that in spite of the large difference in layer spacing among the three surfaces, the key parameters characterizing the response depend only modestly on the crystal orientation.
\end{abstract}

DOI: 10.1103/PhysRevB.66.155413

PACS number(s): 73.20.At, 71.45.Gm, 78.68.+m

\section{INTRODUCTION}

The theoretical description of the electronic excitations at metal surfaces has been a longstanding issue. ${ }^{1-4}$ Regarding simple metals, major progress was achieved by use of the jellium model of Lang and Kohn. ${ }^{5-7}$ Within the timedependent local density approximation (TDLDA), ${ }^{8,9}$ the linear and nonlinear response of semi-infinite jellium to a weak external field was studied extensively. ${ }^{10-13}$ In contrast, the theoretical understanding of electronic excitations at noblemetal and transition-metal surfaces remains at a more phenomenological level. Among these Ag has attracted a particularly large interest as it exhibits a sharp surface plasmon peak which can be utilized as a source for optical chemical sensors. ${ }^{14}$ Also, the $\mathrm{Ag}$ surface plasmon dispersion with a two-dimensional (2D) wave vector $\mathbf{q}$ is qualitatively different from that of simple metals. ${ }^{15-17}$ To understand these properties, response calculations were performed using simplified models in which the filled $4 d$ shell of $\mathrm{Ag}$ was approximated by point dipoles (dipolium) or by a uniform dielectric medium, whereas the $s p$ electrons are treated within the Drude model or quantum mechanically within the jellium approximation. ${ }^{18-22}$

Performing a TDLDA response calculation for real metal surfaces at a finite frequency $\omega$ is still a very demanding task. The difficulty arises in including a sufficient number of lattice vectors in the surface plane as well as in an accurate treatment of the asymptotic behavior of the induced field deep in the bulk. Localized valence $d$ states add a further source of difficulty in the case of transition metals. To our knowledge, until now first-principles response studies of $\mathrm{Ag}$ surfaces were restricted to the static limit where the calculation is reduced to a ground-state problem. Aers and Inglesfield ${ }^{23}$ investigated the electronic structure of a semiinfinite $\operatorname{Ag}(001)$ surface exposed to a uniform electric field using the embedding method of Inglesfield and co-workers. ${ }^{24,25}$ They explored the shifts of Shockley surface states and the screening charge density as a function of the field strength. A similar calculation was performed by Weinert ${ }^{26}$ for $\mathrm{Ag}(001)$ with use of a full-potential linearized augmented plane wave (FLAPW) method ${ }^{27}$ in the slab geometry.

To achieve a more systematic understanding, in the present work, we perform a first-principles static response calculation for three low-index Ag surfaces. Furthermore, as a very preliminary step toward dynamical response studies, we evaluate the electron-hole pair excitation rate within the quasistatic approximation in which the matrix elements of a screened field at low $\omega$ are replaced by those of a linearscreened field at $\omega=0$. The quasistatic excitation rate thus obtained is directly related to a dimensionless parameter $\xi(\mathbf{q})$ introduced by Persson and Zaremba. ${ }^{28}$ A number of spectroscopic quantities can be expressed in terms of $\xi(\mathbf{q})$, including the friction force exerted on atoms and ions moving on a metal surface. ${ }^{29}$ We investigate to what extent the physical parameters concerning the static and quasistatic response of Ag surfaces are sensitive to crystal orientations and how they are different from the corresponding ones for semiinfinite jellium with the same $s p$ electron density as Ag.

The plan of the present paper is as follows. In Sec. II we discuss the ground-state and static response properties of the $\operatorname{Ag}(111), \operatorname{Ag}(001)$, and $\operatorname{Ag}(110)$ surfaces. Section III begins with a brief review of the quasistatic response theory. Then, we present calculated results of the electron-hole pair excitation rate in the quasistatic limit. A summary is given in Sec. IV. We use Hartree atomic units throughout this paper unless otherwise stated.

\section{GROUND-STATE AND STATIC RESPONSE PROPERTIES}

In order to be able to evaluate electron-hole pair excitation rates in the low-frequency limit, it is essential to describe continuous one-electron energy spectra near the Fermi energy $\epsilon_{F}$. Standard slab approximations for the surface calculation are not suitable for this purpose because the energy continuum in the surface normal direction is replaced by discrete levels. In the present work we treat truly semiinfinite Ag surfaces within the local density approximation in density-functional theory ${ }^{30}$ using the embedding technique of Inglesfield. ${ }^{24}$ In this method one first generates the embedding potential of a semi-infinite substrate for a given set of energy mesh points $(\boldsymbol{\epsilon})$ and $2 \mathrm{D}$ wave vectors $(\mathbf{k})$. Next a self-consistent Green-function calculation is performed in the embedded surface region to determine the charge density and the one-electron effective potential. We consider the 


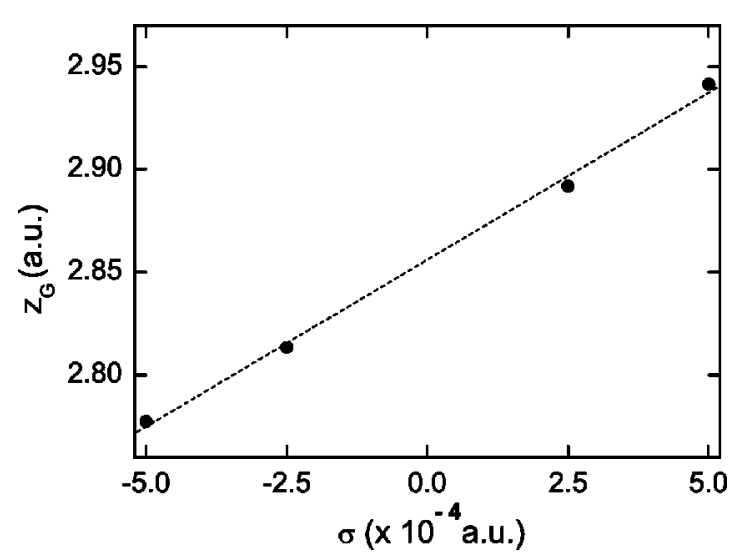

FIG. 1. Centroid of the induced charge density, $z_{G}$, as a function of surface charge $\sigma$ for $\operatorname{Ag}(001) . z_{G}$ is measured relative to the outermost Ag plane. We use the convention that positive $\sigma$ implies a surplus of electrons at the surface.

(111), (001), and (110) crystal surfaces of Ag. The two outermost $\mathrm{Ag}$ atomic layers are included in the embedded surface region, where the spacing between the neighboring $\mathrm{Ag}$ layers is chosen for simplicity as a bulk value. Indeed the atomic relaxations on the (111) and (001) surfaces of $\mathrm{Ag}$ are known to be negligibly small, although the layer spacing between the top and second layers is reduced by $\sim 7 \%$ for the case of $\mathrm{Ag}(110) .{ }^{31} \mathrm{~A}$ uniform electric field perpendicular to the surface can be incorporated as a boundary condition of the Coulomb potential on the vacuum side. The strength of the applied field $E_{a p}$ is related to the induced charge per unit area, $\sigma$, via $E_{a p}=2 \pi \sigma$. More details of our computational method, which combines the embedding approach and the FLAPW technique, were described in Refs. 32 and 33.

For each Ag surface we perform self-consistent electronic-structure calculations for several values of $\sigma$ $\left(|\sigma| \leqslant 5 \times 10^{-4}\right.$ a.u.). Figure 1 shows the calculated centroid of the induced electron density, $z_{G}$, as a function of $\sigma$ for $\mathrm{Ag}(001)$. The points can be fitted by a linear function, which indicates that the electron density in the present $\sigma$ range can be accurately described as

$$
n(\mathbf{r}, \sigma)=n_{0}(\mathbf{r})+\sigma n_{1}(\mathbf{r})+\sigma^{2} n_{2}(\mathbf{r})
$$

where $n_{0}(\mathbf{r}), n_{1}(\mathbf{r})$, and $n_{2}(\mathbf{r})$ are the ground-state, linearinduced, and second-order induced electron densities, respectively. $n_{1}(\mathbf{r})$ has a unit weight per area when averaged over space, while $n_{2}(\mathbf{r})$, with a dipolar character, possesses no net charge. In the same way, the one-electron potential may be expanded as

$$
V(\mathbf{r}, \sigma)=V_{0}(\mathbf{r})+\sigma V_{1}(\mathbf{r})+\sigma^{2} V_{2}(\mathbf{r}) .
$$

From Eq. (1), $n_{1}(\mathbf{r})$ and $n_{2}(\mathbf{r})$ are obtained as ${ }^{34-36}$

$$
\begin{gathered}
n_{1}(\mathbf{r})=\frac{1}{2 \sigma_{0}}\left[n\left(\mathbf{r}, \sigma_{0}\right)-n\left(\mathbf{r},-\sigma_{0}\right)\right], \\
n_{2}(\mathbf{r})=\frac{1}{2 \sigma_{0}^{2}}\left[n\left(\mathbf{r}, \sigma_{0}\right)+n\left(\mathbf{r},-\sigma_{0}\right)-2 n(\mathbf{r}, 0)\right],
\end{gathered}
$$

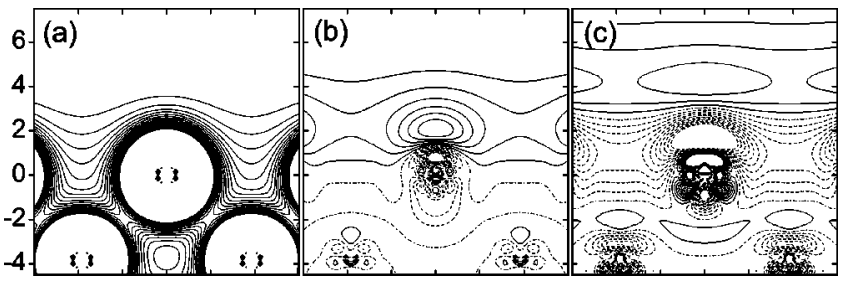

FIG. 2. Contour maps of (a) $n_{0}(\mathbf{r})$, (b) $n_{1}(\mathbf{r})$, and (c) $n_{2}(\mathbf{r})$ on a vertical cut plane containing atoms in the top layer and their nearest neighbors in the second layer for $\operatorname{Ag}(001)$. Solid, dashed, and dot-dashed lines correspond to positive, negative, and zero values of charge densities, respectively. Contour spacings are 2.5 $\times 10^{-3}, 0.1$, and 4 a.u. from the left to right panels.

where $\sigma_{0}$ is a small number within the range of Fig. 1.

Figure 2 shows the calculated contour maps of $n_{0}(\mathbf{r})$, $n_{1}(\mathbf{r})$, and $n_{2}(\mathbf{r})$ for $\mathrm{Ag}(001)$ on a vertical cut plane containing atoms in the first layer and their nearest neighbors in the second layer. Solid, dashed, and dot-dashed contours correspond to positive, negative, and zero values, respectively. The linear-induced charge $n_{1}(\mathbf{r})$ is characterized by a major screening charge formed on the vacuum side of the top-layer atoms and Friedel oscillations toward the interior of the metal. The complex contour patterns in the Ag core region in Fig. 2(b) are very similar to those obtained by Aers and Inglesfield. ${ }^{23}$ The second-order induced charge $n_{2}(\mathbf{r})$ was not shown in their paper. $n_{2}(\mathbf{r})$, plotted in Fig. 2(c), consists of a broad positive peak extended further toward the vacuum than the main peak of $n_{1}(\mathbf{r})$, a negative peak just above the $\mathrm{Ag}$ atoms, and Friedel oscillations toward the bulk. Apart from the complex contours inside the $\mathrm{Ag}$ core region and the lateral corrugation of the charge density, these features are very similar to those obtained for clean jellium surfaces ${ }^{34}$ and for alkali-metal overlayers on jellium. ${ }^{36}$

To examine the crystal-orientation dependence, we plot planar averages of the linear-induced and nonlinear-induced charge densities for the $\mathrm{Ag}(111),(001)$, and (110) surfaces in Fig. 3. We choose the $z$ axis as the surface normal pointing toward the vacuum. In spite of the large difference in interlayer spacing $d$, which equals $\sqrt{6} a / 3, a / \sqrt{2}$, and $a / 2$ for these three surfaces, respectively $(a=5.43$ a.u. denotes the nearest-neighbor Ag-Ag bond length), the planar averages of $n_{1}(\mathbf{r})$ and $n_{2}(\mathbf{r})$ in Fig. 3 look qualitatively very similar. For a more quantitative comparison we evaluate two important parameters. The first is the centroid of the planar average of $n_{1}(\mathbf{r}), z_{1}$, which gives the position of the static image plane. ${ }^{7}\left(z_{1}\right.$ coincides with $z_{G}$ in the limit $\sigma=0$. $)$ The second parameter is the dipole moment associated with the planar average of $n_{2}(\mathbf{r}), p_{2}$, which is proportional to the nonlinear polarizability $\chi_{z z z}(\omega)$ in the limit of $\omega \rightarrow 0 . \chi_{z z z}(\omega)$ can be measured from the intensity of the optical second harmonic generation. ${ }^{11,34}$

Table I lists the calculated $z_{1}$ and $p_{2}$ values of the three surfaces together with their layer spacings $d$, where $z_{1}$ is measured relative to the position of the top atomic plane. For completeness we also give the calculated work functions $\Phi$ of the three Ag surfaces. The calculated $\Phi$ 's for the (111) and (001) surfaces are slightly smaller than those in Ref. 32, because in the present work we employed an improved ver- 


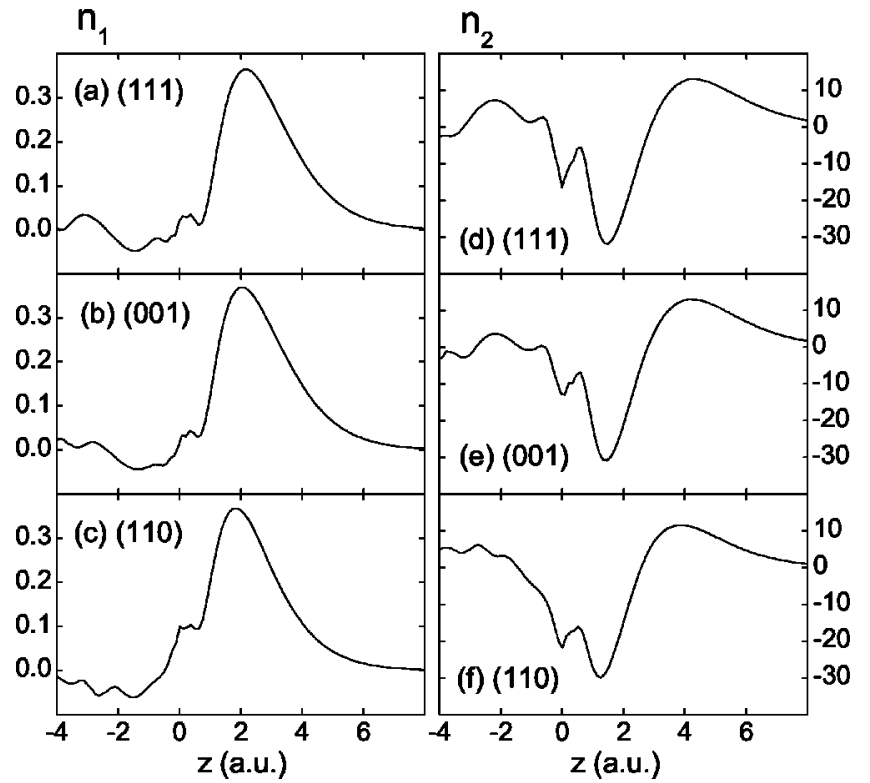

FIG. 3. Planar average of the linear-induced charge $n_{1}(\mathbf{r})$ and nonlinear-induced charge $n_{2}(\mathbf{r})$ for the three low-index Ag surfaces. The origin of the $z$ axis is chosen as the outermost Ag plane.

sion of the embedding potential generated from the complex band structure of a bulk Ag. ${ }^{33}$ Differently from previous experimental and theoretical works, ${ }^{37,38}$ the work functions for the (001) and (110) surfaces do not follow the Smoluchowski ${ }^{39}$ trend. This may be related partly to the accuracy of the embedding potential for the (110) surface, which will be discussed later, and partly to the very small but non-zero (of the order of 0.01 electrons per unit cell) excess charge in the embedded surface region.

It is seen that $z_{1}$ increases with the increasing layer spacing. However, as may be expected from Fig. 3, the ratio between $z_{1}$ of $\operatorname{Ag}(111)$ and that of $\operatorname{Ag}(110), 1.24$, is much smaller than 1.63 , the corresponding ratio of the interlayer spacing of the two surfaces. In contrast to the variation of $z_{1}$ with layer spacing, the work function, which is determined by the dipole moment of the laterally averaged ground state density $n_{0}$, does not rise monotonically with $d$ but has a minimum for $\operatorname{Ag}(001)$. This shows that the induced density $n_{1}$, which probes primarily the states near the Fermi energy, is differently influenced by the crystal structure than the ground state density.

The $5 s$ electrons of Ag correspond to the electron density of jellium with $r_{s}=3$. According to Weber and Liebsch, ${ }^{34}$

TABLE I. Parameters characterizing the static and quasistatic response of three Ag surfaces.

\begin{tabular}{lccc}
\hline \hline & $(111)$ & $(001)$ & $(110)$ \\
\hline$d$ & 4.43 & 3.84 & 2.72 \\
$z_{1}$ & 2.96 & 2.86 & 2.39 \\
$p_{2}$ & 169 & 162 & 157 \\
$\xi(0)$ & 0.94 & 1.12 & 0.96 \\
$\Phi(\mathrm{eV})$ & 4.90 & 4.71 & 4.79 \\
\hline \hline
\end{tabular}

the linear-induced charge of the jellium surface with $r_{s}=3$ has its centroid shifted by 1.35 a.u. outward from the edge of the positive charge background. Assuming that the Ag top layer is located by $d / 2$ inward from this edge, we obtain $z_{1}$ $=3.56,3.27$, and 2.71 a.u. for the (111), (001), and (110) surfaces, respectively. Except for the (110) surface, these values are too large. The discrepancy arises because the jellium surface with $r_{s}=3$ underestimates the work function of $\mathrm{Ag}$ by more than $1 \mathrm{eV}$, and also because jellium cannot describe the $s-d$ hybridization. With a stabilized jellium model ${ }^{40}$ in which a constant attractive potential is added in the interior of jellium, the induced-charge centroid is moved only slightly inward (1.12 a.u. above the jellium edge $\left.{ }^{41}\right)$. However, if the attractive potential is adjusted to match the calculated work function of Ag, this inward shift is much larger. The centroid is then located about 0.3 a.u. above the jellium edge, so that its distance $z_{1}$ from the atomic plane becomes even smaller than in our three-dimensional calculation. Aers and Inglesfield ${ }^{23}$ obtained $z_{1}=2.89$ a.u. for the $\operatorname{Ag}(001)$ surface, which is in very good agreement with the present result.

According to Table I, the nonlinear induced dipole moments $p_{2}$ also rise with increasing layer spacing. Nevertheless, the differences between the three surfaces are remarkably small. Since the nonlinear-induced density $n_{2}(\mathbf{r})$ is more extended toward the vacuum than $n_{1}(\mathbf{r})$, it is less sensitive to the local atomic configuration and the layer spacing. Also, it is evidently not the case that $p_{2}$ is largest for the surface with the lowest work function. Such a simple relationship holds only for the one-dimensional jellium model. As in the case of $z_{1}$, the three-dimensional crystal structure influences the nonlinear surface response which is extremely sensitive to the electronic states near the Fermi level in a quite different manner than the ground state density.

In previous calculations, $p_{2}$ for $\operatorname{Ag}(001)$ was estimated as 110 a.u. (Ref. 23) and as 180 a.u. (Ref. 26). Thus, our result is closer to the latter. The calculated $p_{2}$ values for jellium and stabilized jellium with $r_{s}=3$ were 365 and 321 a.u., respectively, ${ }^{34,41}$ which are much larger than the corresponding ones of real Ag surfaces.

\section{ELECTRON-HOLE PAIR EXCITATION RATE}

The electronic density induced by a static electric field can be used to evaluate the dynamical response behavior of Ag surfaces in the limit of low frequencies. To establish this relationship let us apply to the system an external field with frequency $\omega$ and 2D wave vector $\mathbf{q}$,

$$
\phi_{\text {ext }}(\mathbf{r}, \omega)=-\frac{2 \pi}{|\mathbf{q}|} e^{i \mathbf{q} \cdot \mathbf{x}} e^{|\mathbf{q}| z} .
$$

The linear-induced electron density can be expressed as

$$
n_{1}(\mathbf{r}, \omega)=\sum_{\mathbf{g}} n_{1}(\mathbf{q}+\mathbf{g}, \omega, z) e^{i(\mathbf{q}+\mathbf{g}) \cdot \mathbf{x}},
$$

where $\mathbf{g}$ stands for reciprocal lattice vectors according to the $2 \mathrm{D}$ translational symmetry. In the vacuum region, the linearinduced field due to $n_{1}(\mathbf{r}, \omega)$ can be written as 


$$
\begin{aligned}
\phi_{\text {ind }}(\mathbf{r}, \omega)= & \frac{2 \pi}{|\mathbf{q}|} g(\mathbf{q}, \omega) e^{i \mathbf{q} \cdot \mathbf{x}} e^{-|\mathbf{q}| z} \\
& +\sum_{\mathbf{g} \neq 0} \phi_{\text {ind }}(\mathbf{q}+\mathbf{g}, \omega, z) e^{i(\mathbf{q}+\mathbf{g}) \cdot \mathbf{x}},
\end{aligned}
$$

where $g(\mathbf{q}, \omega)$ is a dimensionless surface response function introduced by Persson and Andersson ${ }^{42}$ for semi-infinite flat jellium. In the present case the second term is associated with the lattice structure in the plane. $g(\mathbf{q}, \omega)$ is expressed in terms of $n_{1}(\mathbf{r}, \omega)$ as

$$
\begin{aligned}
g(\mathbf{q}, \omega) & =\int_{-\infty}^{+\infty} d z e^{|\mathbf{q}| z} n_{1}(\mathbf{q}, \omega, z) \\
& =-\frac{|\mathbf{q}|}{2 \pi A} \int d \mathbf{r} \phi_{\mathrm{ext}}^{*}(\mathbf{r}, \omega) n_{1}(\mathbf{r}, \omega),
\end{aligned}
$$

where $A$ denotes the surface area. The imaginary part of $g(\mathbf{q}, \omega)$ is related to the electron-hole pair excitation rate per unit area by ${ }^{28}$

$$
w(\mathbf{q}, \omega)=\frac{4 \pi}{|\mathbf{q}|} \operatorname{Im} g(\mathbf{q}, \omega),
$$

with $w(\mathbf{q}, \omega)$ defined by

$$
w(\mathbf{q}, \omega)=\frac{2 \pi}{A} \sum_{i, j}\left(f_{i}-f_{j}\right) \delta\left(\epsilon_{j}-\epsilon_{i}-\omega\right)\left|\left\langle\psi_{i}\left|\phi_{\mathrm{scf}}\right| \psi_{j}\right\rangle\right|^{2} .
$$

In Eq. (9) $\psi_{i}$ stands for a one-electron wave function with energy $\epsilon_{i}, f_{i}=\theta\left(\epsilon_{F}-\epsilon_{i}\right)$ is the Fermi occupation at zero temperature, and $\phi_{\text {scf }}(\mathbf{r}, \omega)=\phi_{\text {ext }}(\mathbf{r}, \omega)+\phi_{\text {ind }}(\mathbf{r}, \omega)$ is the screened linear electric field. Now, we keep q finite and take the limit of $\omega \rightarrow 0$. In this limit, $w(\mathbf{q}, \omega)$ behaves as $w(\mathbf{q}, \omega)=\omega \Xi(\mathbf{q})$ where

$$
\Xi(\mathbf{q})=\frac{2 \pi}{A} \sum_{i, j} \delta\left(\epsilon_{i}-\epsilon_{F}\right) \delta\left(\epsilon_{j}-\epsilon_{F}\right)\left|\left\langle\psi_{i}\left|\phi_{\mathrm{scf}}(\mathbf{r}, 0)\right| \psi_{j}\right\rangle\right|^{2} .
$$

By introducing the Fermi wave number $k_{F}$, the plasma frequency $\omega_{p}$, and a dimensionless function $\xi(\mathbf{q})$ $=\left(k_{F} \omega_{p} / 8 \pi\right) \Xi(\mathbf{q})$, Eq. (8) reads, in the quasistatic limit $\left(\eta=\frac{1}{2} k_{F} \omega|\mathbf{q}|^{-1} \epsilon_{F}^{-1} \ll 1\right)$,

$$
\operatorname{Im} g(\mathbf{q}, \omega)=2 \frac{|\mathbf{q}|}{k_{F}} \frac{\omega}{\omega_{p}} \xi(\mathbf{q}) .
$$

In the above derivation, we took the limit of $\omega \rightarrow 0$ while keeping $\mathbf{q}$ finite. Instead, if we take the limit of $\mathbf{q} \rightarrow 0$ with the constraint $\eta \gg 1$, Eqs. (8) and (9) still hold. However, in this case, $\phi_{\text {scf }}(\mathbf{r}, \omega)$ at low $\omega$ comprises two terms as $\phi_{\text {scf }}(\mathbf{r}, \omega)=\phi_{\text {scf }}(\mathbf{r}, 0)+\phi_{b}(\mathbf{r}, \omega)$, where $\phi_{b}$ denotes the long range part of the screened potential extending over the bulk region. For example, $\phi_{b}=-4 \pi\left(\omega / \omega_{p}\right)^{2}\left(z-z_{1}\right)$ for semiinfinite jellium. As the matrix elements of $z-z_{1}$ diverge as $\left(\epsilon_{j}-\epsilon_{i}\right)^{-2}$, those of $\phi_{b}$ do not vanish even in the limit of $\omega=\epsilon_{j}-\epsilon_{j} \rightarrow 0$. Thus, in this limit, one obtains the same equation as Eq. (11), except that $\xi(\mathbf{q})$ is replaced by $\xi_{s}+\xi_{i}$ $+\xi_{b}$, where each of them is called the surface, interference, and bulk term, respectively. ${ }^{28}$ Among them, $\xi_{s}$ coincides with $\xi(\mathbf{q})$ at $\mathbf{q}=0$.

In the present work we calculate the electron-hole pair excitation rate in the quasi-static limit with the use of Eq. (10). In the limit of $\mathbf{q} \rightarrow 0, \phi_{\text {scf }}(\mathbf{r}, 0)$ in Eq. (10) can be replaced by $V_{1}(\mathbf{r})$ in Eq. (2). $V_{1}(\mathbf{r})$ behaves as $-4 \pi\left(z-z_{1}\right)$ in the asymptotic vacuum region and vanishes in the interior of the metal. Using the Green function, we have

$$
\begin{aligned}
\Xi(0)= & \frac{2}{A \pi} \int d \mathbf{r} d \mathbf{r}^{\prime} \operatorname{Im} G\left(\mathbf{r}, \mathbf{r}^{\prime}, \epsilon_{F}\right) V_{1}\left(\mathbf{r}^{\prime}\right) \\
& \times \operatorname{Im} G\left(\mathbf{r}^{\prime}, \mathbf{r}, \epsilon_{F}\right) V_{1}(\mathbf{r}),
\end{aligned}
$$

where the Green function is calculated using the embedding technique as described in Sec. II. The calculated values of $\xi_{s}=\xi(\mathbf{q}=0)$ for the three Ag surfaces are tabulated in the fourth row of Table I. To facilitate a comparison with the previous jellium calculation, we used the jellium parameters for $r_{s}=3$, i.e., $k_{F}=0.64$ a.u., and $\omega_{p}=0.33$ a.u. in converting $\Xi(0)$ to a dimensionless parameter $\xi(0)$. It is to be noted that this $\omega_{p}$ value does not correspond to the observed plasmon energy of Ag, $3.8 \mathrm{eV}$. According to Liebsch, ${ }^{29} \xi(\mathbf{q})$ for jellium with $r_{s}=3$ is a slowly varying function of $\mathbf{q}$, which starts from 1.32 at $\mathbf{q}=0$, exhibits a broad maximum at $|\mathbf{q}| \sim 0.4$ a.u. and tends to 0 at larger $|\mathbf{q}|$. From Table $I$ it is seen that the jellium with $r_{s}=3$ overestimates $\xi(0)$ of the Ag surfaces by $15 \sim 30 \%$, indicating that energy dissipation takes place on real Ag surfaces less frequently than on jellium. This large difference is primarily due to small work function obtained in the jellium model which makes the overall density profile smoother and more polarizable, and therefore overestimates the rate of electron-hole pair production. Also note that $\xi(0)$ does not follow the monotonic trend of $z_{1}$ and $p_{2}$. Instead, it shows a maximum for $\operatorname{Ag}(001)$, i.e., where the work function is smallest. This trend is similar to the one found for the one-dimensional jellium model where a higher work function implies weaker surface response and therefore smaller $\xi(0) .{ }^{28}$ Despite this similarity, the discussion below reveals the importance of three-dimensional lattice effects on the dynamical response properties of realistic metal surfaces.

Since the Green function is diagonal with respect to $\mathbf{k}$, the integral with respect to the planar components of the coordinates in Eq. (12) can be expressed in terms of the $\mathbf{k}$ integral in the surface Brillouin zone (SBZ) and discrete summation over 2D reciprocal lattice vectors at a given $\mathbf{k}$. If we write $\Xi(0)$ as $\Xi(0)=\int p(\mathbf{k}) d \mathbf{k}, p(\mathbf{k})$ equals the sum of $\left|\left\langle\psi_{i}\left|V_{1}\right| \psi_{j}\right\rangle\right|^{2}$ over all the possible pairs of $\psi_{i}$ and $\psi_{j}$, apart from a constant factor. $\psi_{i}$ at a given $\mathbf{k}$ is specified by the band index and $k_{z}$, the $z$ component of the wave vector of the Bloch state incident on the surface. The number of such states is determined by the projection of the threedimensional Fermi surface onto the SBZ.

The Fermi surface of Ag consists of a single $5 s$ band. Thus, in case of $\operatorname{Ag}(001)$, except for a small portion of the SBZ near the zone boundary, one finds a single $5 s$ state for a given $\mathbf{k}$, and the electron-hole pair excitation with infinitesi- 


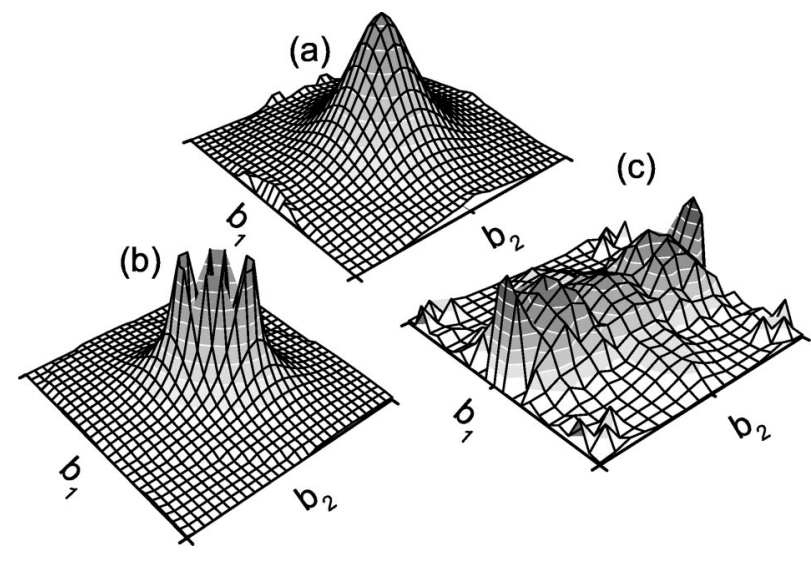

FIG. 4. Distribution of $p(\mathbf{k})$ in the SBZ for (a) $\operatorname{Ag}(001)$, (b) $\operatorname{Ag}(111)$, and (c) $\operatorname{Ag}(110)$ in arbitrary units. Two sides of the horizontal square correspond to two fundamental reciprocal lattice vectors $\mathbf{b}_{1}$ and $\mathbf{b}_{2}$, and the center of the square corresponds to $\mathbf{k}=0$. Note that the actual angle between them is $\pi / 3$ for $\operatorname{Ag}(111)$. Also, $\left|\mathbf{b}_{1}\right| /\left|\mathbf{b}_{2}\right|=\sqrt{2}$ for $\operatorname{Ag}(110)$, where $\mathbf{b}_{1}$ is parallel to the atomic chains of the top-layer $\mathrm{Ag}$ atoms.

mal energy takes place through an infinitesimal shift of $k_{z}$. In Fig. 4(a) we show the calculated $p(\mathbf{k})$ for $\mathrm{Ag}(001)$. It has a large peak at $\mathbf{k}=0$, and decreases rapidly with increasing k. This behavior can be understood within the simple jellium model. That is, at $\mathbf{k}=0$, the one-electron state on the Fermi surface has the largest $k_{z}$. The tail of its wave function is extended toward the vacuum and has the largest overlap with the induced potential $V_{1}$. With increasing $\mathbf{k}$, the corresponding $k_{z}$ value of the state having $\epsilon_{F}$ decreases and the tail of its electron wave function decays more rapidly toward the vacuum. This results in a rapid decrease of the matrix element of $V_{1}$ with increasing $\mathbf{k}$.

On the other hand, $p(\mathbf{k})$ for $\operatorname{Ag}(111)$ in Fig. 4(b) highlights the deviation of the Fermi surface of $\mathrm{Ag}$ from that of jellium. As is well known, the Fermi surface of $\mathrm{Ag}$ is connected in the [111] direction, and within the circular region around $\mathbf{k}=0$, which corresponds to the projection of the neck of the Fermi surface onto the SBZ of $\operatorname{Ag}(111)$, there is no state having $\epsilon_{F}$. Within this energy-gap region, $p(\mathbf{k})$ vanishes identically, leading to a volcanolike shape of $p(\mathbf{k})$. This may explain why the calculated $\xi(0)$ for $\operatorname{Ag}(111)$ is considerably smaller than that of $\operatorname{Ag}(001)$.

Figure 4(c) shows the calculated $p(\mathbf{k})$ for $\operatorname{Ag}(110)$. It looks more spiky than the two previous panels. In fact, with the decreasing interlayer spacing between two atomic layers, calculating the embedding potential of the substrate at high accuracy becomes more difficult. While ground-state properties like $z_{1}$ and $p_{2}$ are rather insensitive to the quality of the embedding potential, the matrix elements entering into $p(\mathbf{k})$ are found to be very sensitive to the embedding potential. Hence, $\xi(0)$ in Table I for $\operatorname{Ag}(110)$ might have a larger numerical error than the other two. Apart from this numerical difficulty, one still notices a qualtitatively different behavior in $p(\mathbf{k})$ for $\operatorname{Ag}(110)$. In contrast to the other two densely packed surfaces, $p(\mathbf{k})$ for $\operatorname{Ag}(110)$ is more anisotropic and does not exhibit a monotonic decay in the direction of $\mathbf{b}_{2}$ which is perpendicular to the atomic chains consisting of top-layer $\mathrm{Ag}$ atoms. This may be interpreted as evidence that the electronic properties of this surface are of more 2D nature.

\section{SUMMARY}

We studied the electronic structure of three low-index Ag surfaces under application of a uniform electric field oriented perpendicular to the surface, using a surface-embedded Green-function code that we developed recently. We demonstrated that our method is accurate enough to determine not only the linear-induced electron density but also the secondorder nonlinear-induced electron density. In addition to those static response properties, we estimated the electron-hole pair excitation rate in the quasistatic limit which is equivalent to a surface response function $\xi(\mathbf{q})$ at $\mathbf{q}=0$. We discussed the crystal-face dependence of the response properties as well as how they are different from the corresponding ones of jellium with the same $s p$ electron density. The main result is that the central quantities characterizing the response depend differently on crystal orientation, according to their specific sensitivity to the range of electronic states which they primarily sample. In particular, the response properties do not follow the variation of the work function with crystal orientation.

As stated in Sec. I, performing a first-principles dynamical response calculation for semi-infinite crystal surfaces still seems too difficult. The easiest extension of the present study may be to determine $\xi(\mathbf{q})$ at finite $\mathbf{q}$. This quantity can be calculated from formula (12) by replacing $V_{1}(\mathbf{r})$ by a screened field $\phi_{\text {scf }}(\mathbf{q}, 0)$ corresponding to finite $\mathbf{q}$. If $\mathbf{q}$ is chosen as commensurate as the surface unit cell, the calculation of $\phi_{\mathrm{scf}}(\mathbf{q}, 0)$ is reduced to a standard ground-state electronic structure calculation using a large supercell.
${ }^{1}$ A. Liebsch, Electronic Excitations at Metal Surfaces (Plenum, New York, 1997).

${ }^{2}$ H. Raether, Surface Plasmon (Springer, Berlin, 1988).

${ }^{3}$ Electromagnetic Surface Modes, edited by A. D. Boardman (Wileys, New York, 1982).

${ }^{4}$ P. J. Feibelman, Prog. Surf. Sci. 12, 287 (1982).

${ }^{5}$ N. D. Lang and W. Kohn, Phys. Rev. B 1, 4555 (1970).

${ }^{6}$ N. D. Lang and W. Kohn, Phys. Rev. B 3, 1215 (1971).

${ }^{7}$ N. D. Lang and W. Kohn, Phys. Rev. B 7, 3541 (1973).

\footnotetext{
${ }^{8}$ A. Zangwill and P. Soven, Phys. Rev. A 21, 1561 (1980).

${ }^{9}$ G. D. Mahan and K. R. Subbaswamy, Local Density Theory of Polarizability (Plenum, New York, 1990).

${ }^{10}$ A. Liebsch, Phys. Rev. B 36, 7378 (1987).

${ }^{11}$ A. Liebsch, Phys. Rev. Lett. 61, 1233 (1988).

${ }^{12}$ A. Liebsch, Phys. Rev. B 40, 3421 (1988).

${ }^{13}$ K. D. Tsuei, E. W. Plummer, A. Liebsch, K. Kempa, and P. Bakshi, Phys. Rev. Lett. 64, 44 (1990).

${ }^{14}$ B. Rothenhaeusler and W. Knoll, Nature (London) 332, 615
} 
(1988).

${ }^{15}$ S. Suto, K. Tsuei, E. W. Plummer, and E. Burstein, Phys. Rev. Lett. 63, 2590 (1989).

${ }^{16}$ M. Rocca and U. Valbusa, Phys. Rev. Lett. 64, 2398 (1990).

${ }^{17}$ G. Lee, P. T. Sprunger, E. W. Plummer, and S. Suto, Phys. Rev. Lett. 67, 3198 (1991).

${ }^{18}$ J. Tarriba and W. L. Mochán, Phys. Rev. B 46, 12902 (1992).

${ }^{19}$ C. Lopéz-Bastidas and W. L. Mochán, Phys. Rev. B 60, 8348 (1999).

${ }^{20}$ C. Lopéz-Bastidas, A. Liebsch, and W. L. Mochán, Phys. Rev. B 63, 165407 (2001).

${ }^{21}$ A. Liebsch, Phys. Rev. Lett. 71, 145 (1993).

${ }^{22}$ A. Liebsch, Phys. Rev. B 57, 3803 (1998).

${ }^{23}$ G. C. Aers and J. E. Inglesfield, Surf. Sci. 217, 367 (1989).

${ }^{24}$ J. E. Inglesfield, J. Phys. C 14, 3795 (1981).

${ }^{25}$ J. E. Inglesfield and G. A. Benesh, Phys. Rev. B 37, 6682 (1988).

${ }^{26} \mathrm{M}$. Weinert (private communication).

${ }^{27}$ D. J. Singh, Planewaves, Pseudopotentials and the LAPW Method (Kluwer, Boston, 1994).
${ }^{28}$ B. N. J. Persson and E. Zaremba, Phys. Rev. B 31, 1863 (1985).

${ }^{29}$ A. Liebsch, Phys. Rev. B 55, 13263 (1997).

${ }^{30}$ W. Kohn and L. J. Sham, Phys. Rev. 140, A1133 (1965).

${ }^{31}$ M. Lindroos, C. J. Barnes, M. Valden, and D. A. King, Surf. Sci. 218, 269 (1989).

${ }^{32}$ H. Ishida, Phys. Rev. B 63, 165409 (2001).

${ }^{33}$ D. Wortmann, H. Ishida, and S. Blügel, Phys. Rev. B 65, 165103 (2002).

${ }^{34}$ M. G. Weber and A. Liebsch, Phys. Rev. B 35, 7411 (1987).

${ }^{35}$ M. G. Weber and A. Liebsch, Phys. Rev. B 36, 6411 (1987).

${ }^{36}$ H. Ishida and A. Liebsch, Phys. Rev. B 42, 5505 (1990).

${ }^{37}$ M. Methfessel, D. Hennig, and M. Scheffler, Phys. Rev. B 46, 4816 (1992).

${ }^{38}$ C. J. Fall, N. Binggeli, and A. Baldereschi, Phys. Rev. B 61, 8489 (2000).

${ }^{39}$ R. Smoluchowski, Phys. Rev. 60, 661 (1941).

${ }^{40}$ H. B. Shore and J. H. Rose, Phys. Rev. Lett. 66, 2519 (1991).

${ }^{41}$ A. Kiejna, Surf. Sci. 331-333, 1167 (1995).

${ }^{42}$ B. N. J. Persson and S. Andersson, Phys. Rev. B 30, 4382 (1984). 